\title{
Fixation Strength of Caudal Pedicle Screws after Posterior Lumbar Interbody Fusion with the Modified Cortical Bone Trajectory Screw Method
}

\author{
Hironobu Sakaura ${ }^{1}$, Toshitada Miwa ${ }^{1}$, Tomoya Yamashita ${ }^{2}$, Yusuke Kuroda ${ }^{1}$, Tetsuo Ohwada ${ }^{1}$ \\ ${ }^{1}$ Department of Orthopaedic Surgery, Kansai Rosai Hospital, Amagasaki, Japan \\ ${ }^{2}$ Department of Orthopaedic Surgery, Osaka Rosai Hospital, Osaka, Japan
}

Study Design: Clinical case series.

Purpose: In the posterior lumbar interbody fusion (PLIF) procedure in our institute, the cephalad screw trajectory follows a mediolateral and caudocephalad directed path according to the original cortical bone trajectory (CBT) method. However, the starting point of the caudal screw is at the medial border of the pedicle on an articular surface of the superior articular process, and the trajectory takes a mediolateral path parallel to the cephalad endplate. The incidence of caudal screw loosening after PLIF with this modified CBT screw method was investigated, and significant risk factors for caudal screw loosening were evaluated.

Overview of Literature: A biomechanical study of this modified caudal screw trajectory using the finite element method reported about a $20 \%$ increase in uniaxial yield pullout load compared with the traditional trajectory. However, there has been no clinical study concerning the fixation strength of this modified caudal screw trajectory.

Methods: The subjects were 193 consecutive patients who underwent single-level PLIF with modified CBT screw fixation. Caudal screw loosening was checked in computed tomography at 6 months after surgery, and screw loosening was defined as a radiolucency of $1 \mathrm{~mm}$ or more at the bone-screw interface.

Results: The incidence of caudal screw loosening after lumbosacral PLIF (46.2\%) was significantly higher than that after floating PLIF (6.0\%). No significant differences in sex, brand of the instruments, and diameter and length of the caudal screw were evident between patients with and without caudal screw loosening. Patients with caudal screw loosening were significantly older at the time of surgery than patients without caudal screw loosening.

Conclusions: Fixation strength of the caudal screw after floating PLIF with this modified CBT screw technique was sufficiently acceptable. Fixation strength after the lumbosacral procedure was not.

Keywords: Posterior lumbar interbody fusion; Less-invasive approach; Cortical bone trajectory; Caudal screw loosening; Risk factors

\section{Introduction}

As a new alternative strategy to obtain improved pedicle screw fixation in the lumbar spine, a cortical bone trajectory (CBT) screw technique was advocated by Santoni et al. [1] in 2009. Several biomechanical studies have

Received Oct 21, 2015; Revised Oct 30, 2015; Accepted Nov 2, 2015

Corresponding author: Hironobu Sakaura

Department of Orthopaedic Surgery, Kansai Rosai Hospital,

3-1-69 Inabaso, Amagasaki, Hyogo 660-8511, Japan

Tel: +81-6-6416-1221, Fax: +81-6-6419-1870, E-mail: sakaura04061023@yahoo.co.jp 
demonstrated the non-inferior mechanical properties of the CBT screw in cadaveric lumbar specimens [1-3]. Moreover, screw insertion through a mediocaudal starting point helps limit dissection of the superior facet joints and reduces muscle dissection and retraction. During the posterior lumbar interbody fusion (PLIF) procedure with CBT screw fixation in our institute, the cephalad screw trajectory follows a mediolateral and caudocephalad directed path according to the original method [1]. However, the starting point of the caudal screw is at the medial border of the pedicle on an articular surface of the superior articular process, and the trajectory takes a mediolateral path parallel to the cephalad endplate to minimize approach-related damage. A biomechanical study of this modified caudal screw trajectory using the finite element method reported about a $20 \%$ increase in uniaxial yield pullout load compared with the traditional trajectory [4].

To elucidate the fixation strength of caudal screws in vivo, the incidence of caudal screw loosening after singlelevel PLIF with this modified CBT screw method was investigated, and significant risk factors for caudal screw loosening were evaluated.

\section{Materials and Methods}

\section{Patients}

The subjects were 193 consecutive patients (103 men, 90 women; mean age at time of surgery, 68.3 years; age range, 31-85 years) who underwent single-level PLIF with this modified CBT screw technique for unstable degenerative lumbar spinal disorders between October 2011 and December 2013. Etiologies of degenerative lumbar spinal disorders were: degenerative lumbar spondylolisthesis in 128 patients; lumbar spinal stenosis with lateral slippage on an anteroposterior radiograph of the lumbar spine in 39; isthmic spondylolisthesis in 16; and lumbar disc herniation with posterior opening on a flexion lateral radiograph of the lumbar spine in 10. Fused areas were as follows: L4 to L5 in 124 patients; L3 to L4 in 35; L5 to S1 in 26; L2 to L3 in 5; L5 to L6 in 2; and L1 to L2 in 1 . All patients were considered for surgery due to unresponsiveness to conservative treatment, such as medication and/or epidural block. The protocol was approved by the Institutional Review Board of the hospital, and written informed consent was obtained from all participants.

\section{Surgical procedure}

A small skin incision (about $5 \mathrm{~cm}$ ) was made at the fused segment. Dissection of facet joints and capsules supradjacent to the fused segment was limited as much as possible. Posterior neural decompression including laminotomy and partial or total facetectomy was performed. After intervertebral disc materials and cartilaginous end plates were removed, two carbon fiber reinforced polyetheretherketone cages filled with local bone graft were inserted into the intervertebral space, and local bone blocks were inserted lateral or medial to the cages. The cephalad screw trajectory followed a mediolateral and caudocephalad directed path according to the original method [1]. On the other hand, the starting point of the caudal screw was at the medial border of the pedicle on an articular surface of the superior articular process, and the trajectory took a mediolateral path parallel to the cephalad endplate to minimize approach-related damage. The brands of instruments used in the 193 patients were: CD HORIZON SOLERA Spinal System (Medtronic Inc., Memphis, TN, USA) in 113 patients; Small XIA Spinal System (Stryker Japan, Tokyo, Japan) in 57; Expedium 5.5 Spine System (DepuySynthes Inc., Raynham, MA, USA) in 19; and miscellaneous others in 4 . The fused area was stabilized using screws and rods with the application of an appropriate compression force (Figs. 1, 2). All patients wore a thoracolumbosacral orthosis for 3 postoperative months.

\section{Radiologic evaluations}

Caudal screw loosening was checked in multiplanar reconstruction computed tomography (MPR-CT) at 6 months after surgery, and screw loosening was defined as a radiolucency of $1 \mathrm{~mm}$ or more at the bone-screw interface (Figs. 1, 2) [5].

\section{Risk factors for caudal screw loosening}

Age at time of surgery, gender, etiologies of degenerative lumbar spinal disorders, operated level, brand of the instruments, and diameter and length of the caudal screw were analyzed to identify risk factors for caudal screw loosening. 



Fig. 1. Radiographs and multiplanar reconstruction computed tomography of the lumbar spine at 6 months after surgery in a 56-year-old man who underwent less-invasive posterior lumbar interbody fusion with our cortical bone trajectory screw fixation for $L 4$ degenerative spondylolisthesis. Computed tomography shows no caudal screw loosening at L5. (A) Anteroposterior view. (B) Lateral view. (C) Axial view. (D) Coronal view.
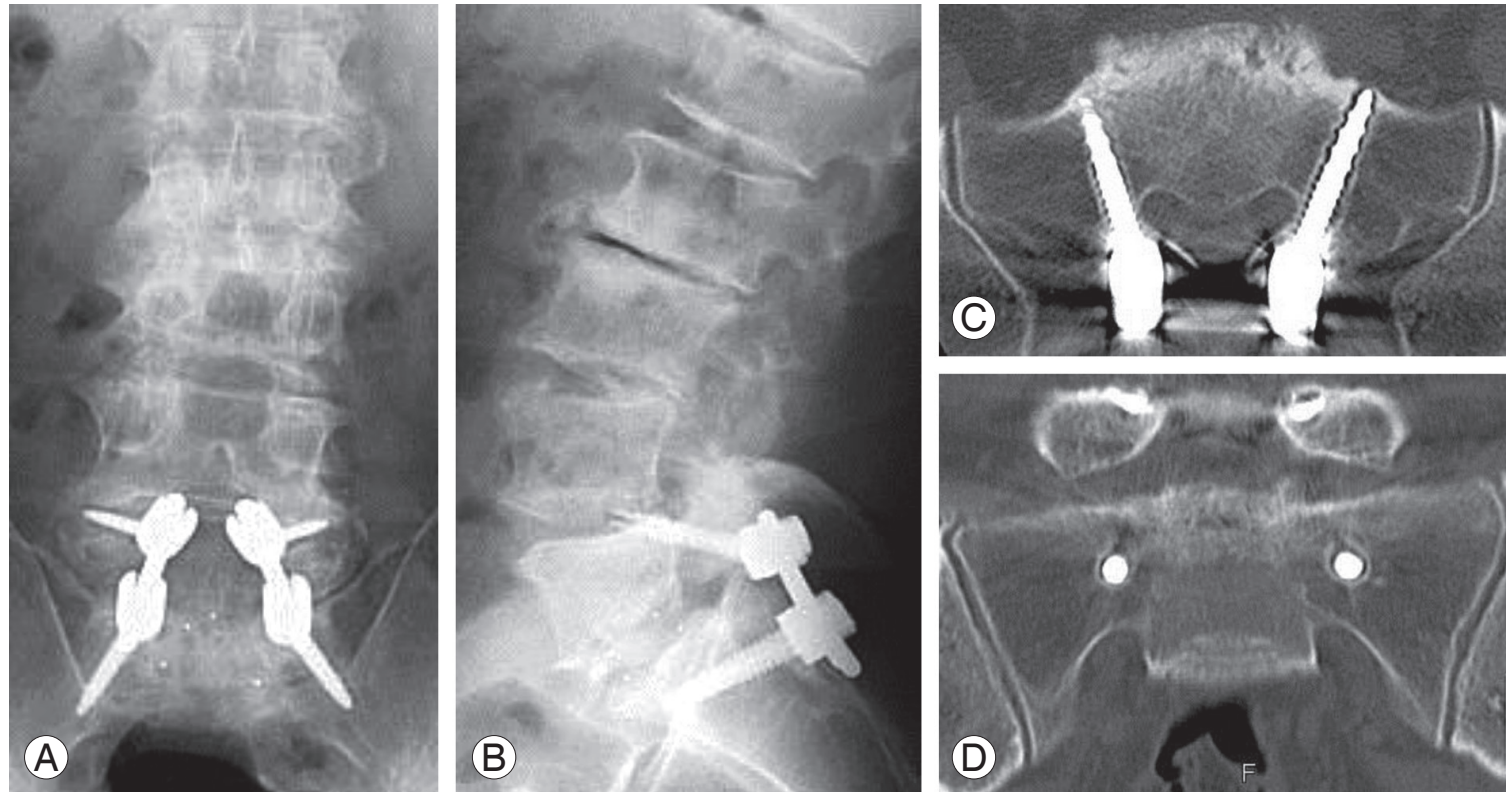

Fig. 2. Radiographs and multiplanar reconstruction computed tomography of the lumbar spine at 6 months after surgery in a 61 -year-old man who underwent less-invasive posterior lumbar interbody fusion with our cortical bone trajectory screw fixation for L5 isthmic spondylolisthesis Computed tomography shows bilateral caudal screw loosening at S1. (A) Anteroposterior view. (B) Lateral view. (C) Axial view. (D) Coronal view.

\section{Statistical analyses}

The unpaired $t$-test, Kruskal-Wallis test, Mann-Whitney
U test with Bonferroni correction and Fisher's exact probability test were used for statistical analyses with JMP 5.0.1 software (SAS Institute, Cary, NC, USA) as appropriate. 
Values of $p<0.05$ were considered significant.

\section{Results}

\section{Caudal screw loosening}

Overall, 44 of 386 caudal screws (11.4\%) in 29 of 193 patients (15.0\%) loosened at 6 months after surgery. Caudal screw loosening was found in none of the 2 screws at L2; none of the 10 screws at L3; 4 of the 70 screws at L4; 16 of the 248 screws at L5; none of the 4 screws at L6; and 24 of the 52 screws at $S 1$. The incidence of caudal screw loosening was significantly higher at $\mathrm{S} 1$ than at other vertebral levels (Mann-Whitney U test with Bonferroni correction; $p<0.01$ ) (Table 1).

\section{Risk factors for caudal screw loosening}

The incidence of caudal screw loosening was significantly higher after lumbosacral PLIF (24/52 screws, 46.2\%; $13 / 26$ patients, 50.0\%) than after PLIF at the segment of the mobile lumbar spine (from L1/2 to L5/L6, floating PLIF) (20/334 screws, $6.0 \% ; 16 / 167$ patients, $9.6 \%$, Fisher's exact probability test; $p<0.01)$. No significant differences in gender, brand of the instruments, and diameter and length of

Table 1. Caudal screw loosening at each level

\begin{tabular}{lc} 
Vertebral level & Caudal screw loosening (\%) \\
L2 & $0 / 2(0)$ \\
L3 & $0 / 10(0)$ \\
L4 & $4 / 70(5.7)$ \\
L5 & $16 / 248(6.5)$ \\
L6 & $0 / 4(0)$ \\
S1 & $24 / 52(46.2)^{\text {a) }}$ \\
\hline
\end{tabular}

a) Significantly higher than at other levels $(p<0.01)$. the caudal screw were evident between patients with and without caudal screw loosening (Fisher's exact probability test, Kruskal-Wallis test, unpaired $t$-test; $p>0.05$ ) (Table $2)$. In contrast, the incidence of caudal screw loosening in patients with isthmic spondylolisthesis (13/32 screws, $40.6 \%$; 7/16 patients, $43.8 \%$ ) was significantly higher than that in patients with other etiologies (31/354 screws, $8.8 \% ; 22 / 177$ patients, $12.4 \%$, Fisher's exact probability test; $p<0.05)$. Patients with caudal screw loosening were significantly older at time of surgery than patients without caudal screw loosening (unpaired $t$-test; $p<0.01$ ) (Table 2).

\section{Discussion}

A CBT screw technique was advocated by Santoni et al. [1] in 2009 as a new alternative strategy to improve pedicle screw fixation in the lumbar spine. Several biomechanical studies have demonstrated the non-inferior mechanical properties of a construct using CBT screw fixation in cadaveric lumbar specimens $[2,3]$. Some researchers reported that $\mathrm{CBT}$ screw-rod fixation provides about the same stability as traditional pedicle screw-rod fixation, regardless of the presence of interbody support [2]. Others reported that a CBT screw-rod construct has significantly superior resistance to craniocaudal toggling displacement compared with a traditional pedicle screw-rod construct [3]. In addition, screw insertion through a mediocaudal starting point enables less-invasive posterior lumbar fixation by limiting dissection of the superior facet joints and reducing muscle dissection and retraction. However, whereas CBT screwing at the caudal level of fusion according to the original method [1] requires paravertebral muscle dissection at the inferior lamina, caudal screws can be inserted using this modified technique without any further caudal paravertebral muscle dissection after posterior decompression, interbody bone grafting, and cranial screw insertion. To minimize approach-related

Table 2. Risk factors for caudal screw loosening

Age at time of surgery $(\mathrm{yr})$
Diameter of the caudal screw $(\mathrm{mm})$
Length of the caudal screw $(\mathrm{mm})$
Values are presented as mean \pm standard deviation.
a) Significantly older than in patients without caudal screw loosening $(p<0.01)$.

$73.1 \pm 7.1^{\text {a) }}$
$67.5 \pm 10.8$ 
damage, we have performed PLIF with this modified CBT screw technique since October 2011. A cadaveric study demonstrated that the CBT screw according to the original method provided a $30 \%$ increase in uniaxial yield pullout load relative to the traditional pedicle screw [1]. On the other hand, a biomechanical study using the finite element analysis showed that the mean resistance against screw pullout for this modified caudal trajectory screw was $1236 \mathrm{~N}$ and $1036 \mathrm{~N}$ for the traditional pedicle screw [4]. However, there has been no clinical study concerning the fixation strength of this modified caudal screw trajectory in vivo so far. Therefore, in order to elucidate the fixation strength of caudal screws in vivo, the incidence of caudal screw loosening after single-level PLIF with this modified CBT screw fixation method was investigated. A clinical and experimental study proved that a radiolucent zone at the bone-screw interface is a good indicator of pedicle screw loosening [6]. Most of the lumbar pedicle screw failures, including screw loosening, reportedly occurred within 6 months after surgery [7]. Based on these results, in the present study, caudal screw loosening was checked on MPR-CT at 6 months after surgery, and screw loosening was defined as a radiolucency of $1 \mathrm{~mm}$ or more at the bone-screw interface [5].

In the present study, 44 of 386 caudal screws (11.4\%) in 29 of 193 patients (15.0\%) loosened. The incidence of caudal screw loosening was $6.0 \%$ of the screws in $9.6 \%$ of the patients after floating PLIF and $46.2 \%$ of the screws in $50.0 \%$ of the patients after lumbosacral PLIF. Concerning traditional pedicle screw fixation, the incidence of screw loosening ranged from $0.6 \%$ to $11 \%$ in a literature review [8]. In the Japanese population, the incidence of screw loosening after single-level PLIF using traditional pedicle screw fixation reportedly ranged from $4.0 \%$ to $12.6 \%$ of the screws and from $2.7 \%$ to $21.2 \%$ of the patients, respectively $[5,9,10]$. On the other hand, a clinical study reported that 5 of the 81 screws $(6.2 \%)$ in 4 of the 20 patients (20\%) loosened at 1 year after lumbar fusion using CBT screw fixation according to the original method [11]. These results indicate that the fixation strength of the caudal screw after floating PLIF with this modified CBT screw technique is sufficiently acceptable, but fixation strength of the caudal screw after lumbosacral PLIF is not.

Similar to the concept of PLIF with this modified CBT screw fixation, Takata et al. [12] recently reported lessinvasive single-level transforaminal lumbar interbody fusion (TLIF) with CBT screws at the cranial level and traditional pedicle screws at the caudal level. However, because they described very short-term (follow-up period, 3 months) outcomes in a very small sample size $(n=6)$ [12], the fixation strength of their caudal traditional pedicle screws is unclear. The caudal screw trajectory with the modified CBT technique reportedly showed about a $20 \%$ increase in uniaxial yield pullout load compared with the traditional trajectory [4], and fixation strength of the caudal screw after floating PLIF with this modified CBT screw technique was acceptable in the present study. Furthermore, this modified caudal screw trajectory seems to be easier to connect with the cranial CBT screw than the caudal traditional pedicle screw. Therefore, as far as floating PLIF, the procedure with this modified CBT screw fixation may be more favorable than the hybrid technique of Takata et al. [12].

Regarding lumbosacral fusion, in the present study, the incidence of caudal screw loosening was significantly higher at S1 (46.2\%) than at other vertebral levels (mean $6.0 \%)$. The incidence of caudal screw loosening in patients with isthmic spondylolisthesis was significantly higher than that in patients with other etiologies. This result is also greatly due to the clinical feature of isthmic spondylolisthesis in that the L5 vertebra is the most frequent site of isthmic spondylolisthesis (in this study, 12 of the 16 patients with isthmic spondylolisthesis underwent lumbosacral PLIF). The lumbosacral junction remains a difficult area to achieve a successful spinal fusion, and sacral screw fixation has been challenging for construct failures such as screw loosening $[13,14]$. Excessive mechanical stress on the lumbosacral junction, the peculiar features of the sacrum, and inadequate sacral bone purchase may result in the failure of sacral screw fixation [15-17]. Since the sacrum contains a confluence of cancellous bone from the first sacral segment vertebral body to the sacral ala, the sacrum does not have a true pedicle of cortical bone ring [18]. Regarding the fixation strength of sacral screw fixation after TLIF or posterolateral fusion, a recent prospective study of 90 consecutive patients showed that sacral screw loosening and lumbosacral instability were more prevalent in patients treated with monocortical sacral screw fixation than in those with tricortical sacral screw fixation [19]. However, since all biomechanical studies on the CBT screw technique have been done with lumbar specimens up to L5 and not with sacrum [1-4], the biomechanical properties of the CBT screw inserted at S1 remain unclear. Given these results, this modified caudal 
screw trajectory, which is one of the monocortical screw purchases, may lead to a significantly higher incidence of caudal screw loosening at S1. To reduce the risk of sacral screw loosening after lumbosacral CBT screw fixation, Matsukawa et al. [20] recently advocated the penetrating S1 endplate screw (PES), which was directed straight forward in the axial plane and angulated cranially in the sagittal plane, penetrating the middle of the sacral endplate. An anatomical and radiological study showed that the anterolateral part of the cranial S1 body was the densest area of the sacral trabecular intersection [21]. In addition, sacral osteoporotic change reportedly resulted in the densest area of trabecular bone consistently observed closer to the cranial endplate of the sacrum [22]. Therefore, in vivo measurements by Matsukawa et al. [20] demonstrated that the insertion torque of the PES was $141 \%$ higher than that of the traditional pedicle screw, and they reported that all 33 screws inserted with their PES technique in 19 patients showed no screw loosening. In addition, another advantage of the PES technique is that this screw trajectory can be easily connected with the cranial CBT screw [20]. These results suggest that the PES technique may be one of the promising solutions to obtain improved fixation strength of the sacral screw and to achieve solid lumbosacral fusion with CBT screw fixation. Based on the poor results of the modified caudal CBT screw technique at $\mathrm{S} 1$ in the present study, we have thus inserted pedicle screws at S1 with the PES technique since publication of this technique in 2014.

Another risk factor for caudal screw loosening in the present study was older age at time of surgery. Lower bone mineral density of the lumbar spine reportedly has a close relation with pedicle screw loosening after PLIF with traditional pedicle screw fixation [10]. Bone quality of the lumbar spine deteriorates with age in both men and women [23]. Therefore, poorer bone quality of the lumbar spine may lead to a higher incidence of caudal screw loosening in older patients.

There are several major limitations in this study. The first is lack of a comparison group using traditional pedicle screw fixation. It may not be helpful to make a direct comparison of the results after PLIF using traditional pedicle screw fixation in the literature with the results after PLIF with this modified CBT screw fixation in this study. The second is lack of data on bone quality of the lumbar spine which is an important potential risk factor of spinal stabilization compromise. The third is lack of data on smoking history which is also one of the other potential risk factors of spinal stabilization compromise.

\section{Conclusions}

Whereas fixation strength of the caudal screw after floating PLIF with this modified CBT screw technique was acceptable, that after lumbosacral procedure was not.

\section{Conflict of Interest}

No potential conflict of interest relevant to this article was reported.

\section{References}

1. Santoni BG, Hynes RA, McGilvray KC, et al. Cortical bone trajectory for lumbar pedicle screws. Spine J 2009;9:366-73.

2. Perez-Orribo L, Kalb S, Reyes PM, Chang SW, Crawford NR. Biomechanics of lumbar cortical screw-rod fixation versus pedicle screw-rod fixation with and without interbody support. Spine (Phila Pa 1976) 2013;38:635-41.

3. Baluch DA, Patel AA, Lullo B, et al. Effect of physiological loads on cortical and traditional pedicle screw fixation. Spine (Phila Pa 1976) 2014;39:E1297-302.

4. Matsukawa K, Yato Y, Imabayashi H, Hosogane N, Asazuma T, Nemoto K. Biomechanical evaluation of the fixation strength of lumbar pedicle screws using cortical bone trajectory: a finite element study. J Neurosurg Spine 2015;23:471-8.

5. Okuyama K, Abe E, Suzuki T, Tamura Y, Chiba M, Sato K. Posterior lumbar interbody fusion: a retrospective study of complications after facet joint excision and pedicle screw fixation in 148 cases. Acta Orthop Scand 1999;70:329-34.

6. Sanden B, Olerud C, Petren-Mallmin M, Johansson $\mathrm{C}$, Larsson S. The significance of radiolucent zones surrounding pedicle screws. Definition of screw loosening in spinal instrumentation. J Bone Joint Surg Br 2004;86:457-61.

7. Mohi Eldin MM, Ali AM. Lumbar transpedicular implant failure: a clinical and surgical challenge and its radiological assessment. Asian Spine J 2014;8:28197.

8. Esses SI, Sachs BL, Dreyzin V. Complications associ- 
ated with the technique of pedicle screw fixation: a selected survey of ABS members. Spine (Phila Pa 1976) 1993;18:2231-8.

9. Okuyama K, Abe E, Suzuki T, Tamura Y, Chiba M, Sato K. Can insertional torque predict screw loosening and related failures? An in vivo study of pedicle screw fixation augmenting posterior lumbar interbody fusion. Spine (Phila Pa 1976) 2000;25:858-64.

10. Okuyama K, Abe E, Suzuki T, Tamura Y, Chiba M, Sato $\mathrm{K}$. Influence of bone mineral density on pedicle screw fixation: a study of pedicle screw fixation augmenting posterior lumbar interbody fusion in elderly patients. Spine J 2001;1:402-7.

11. Sakuma T, Kotani T, Akazawa T, et al. Analysis of risk factor of cortical bone trajectory screw loosening: comparison with conventional pedicle screw loosening. J Spinal Res 2014;5:593.

12. Takata Y, Matsuura T, Higashino K, et al. Hybrid technique of cortical bone trajectory and pedicle screwing for minimally invasive spine reconstruction surgery: a technical note. J Med Invest 2014;61:38892.

13. Balderston RA, Winter RB, Moe JH, Bradford DS, Lonstein JE. Fusion to the sacrum for nonparalytic scoliosis in the adult. Spine (Phila Pa 1976) 1986;11: 824-9.

14. Pashman RS, Hu SS, Schendel MJ, Bradford DS. Sacral screw loads in lumbosacral fixation for spinal deformity. Spine (Phila Pa 1976) 1993;18:2465-70.

15. Carlson GD, Abitbol JJ, Anderson DR, et al. Screw fixation in the human sacrum: an in vitro study of the biomechanics of fixation. Spine (Phila Pa 1976) 1992;17(6 Suppl):S196-203.

16. Leong JC, Lu WW, Zheng Y, Zhu Q, Zhong S. Com- parison of the strengths of lumbosacral fixation achieved with techniques using one and two triangulated sacral screws. Spine (Phila Pa 1976) 1998;23: 2289-94.

17. Smith SA, Abitbol JJ, Carlson GD, Anderson DR, Taggart KW, Garfin SR. The effects of depth of penetration, screw orientation, and bone density on sacral screw fixation. Spine (Phila Pa 1976) 1993;18:100610.

18. Robertson PA, Plank LD. Pedicle screw placement at the sacrum: anatomical characterization and limitations at S1. J Spinal Disord 1999;12:227-33.

19. Orita S, Ohtori S, Eguchi Y, et al. Radiographic evaluation of monocortical versus tricortical purchase approaches in lumbosacral fixation with sacral pedicle screws: a prospective study of ninety consecutive patients. Spine (Phila Pa 1976) 2010;35:E1230-7.

20. Matsukawa K, Yato Y, Kato T, Imabayashi H, Asazuma T, Nemoto K. Cortical bone trajectory for lumbosacral fixation: penetrating S-1 endplate screw technique: technical note. J Neurosurg Spine 2014; 21:203-9.

21. Ebraheim N, Sabry FF, Nadim Y, Xu R, Yeasting RA. Internal architecture of the sacrum in the elderly: an anatomic and radiographic study. Spine (Phila $\mathrm{Pa}$ 1976) 2000;25:292-7.

22. Peretz AM, Hipp JA, Heggeness MH. The internal bony architecture of the sacrum. Spine (Phila $\mathrm{Pa}$ 1976) 1998;23:971-4.

23. Samelson EJ, Christiansen BA, Demissie S, et al. QCT measures of bone strength at the thoracic and lumbar spine: the Framingham Study. J Bone Miner Res 2012;27:654-63. 